\title{
A Method Based on RTO and Selective Acknowledgement for Improving SCTP Protocol Performance in Mobile Ad Hoc Networks
}

\author{
${\text { Mostafa } \operatorname{tabaiyan}^{1} \& \text { Mehdi agha Sarram }}^{1}$ \\ ${ }^{1}$ Department of Computer Engineering, Yazd University, Yazd, Iran \\ Correspondence: Mostafa tabaiyan, Department of Computer Engineering, Yazd University, Yazd, Iran. E-mail: \\ mostafatabaiyan@stu.yazd.ac.ir/Mehdi.sarram@yazd.ac.ir
}

Received: April 12, 2016

Accepted: April 25, 2016

Online Published: June 8, 2016

doi:10.5539/mas.v10n6p238

URL: http://dx.doi.org/10.5539/mas.v10n6p238

\begin{abstract}
With the increasing development of Mobile Ad Hoc Network usage, there will be more need to have a transport protocol with an appropriate throughput in end to end transport. Because of the design nature of TCP protocol for wired Networks, its application in Mobile Ad-hoc Networks causes a reduction in efficiency, performance and throughput.

Protocols such as SCTP with sufficient validity and reliability in data transfer are proposed as solution for increasing throughput. However, in order to provide the above quality, sacrifice factors such as Network balance. In the present paper, two optimized transporting algorithm are introduced. These algorithm act based on retransmission timeout control and selective acknowledgement number for transmissions in SCTP protocol. The offered algorithms using implemented NS-2 simulator and throughput improvement and reduction of packet delay time are compared with former protocols.
\end{abstract}

Keywords: protocol, SCTP, Mobile Ad-hoc Networks, throughput, delay, performance

\section{Introduction}

Considering an increase in Ad-hoc Networks usage, applications such as file transmission and web reviewer, are used in mobile Ad-hoc Networks under TCP Protocol broadly. Since this protocol is designed more for wired networks, and it is not appropriate for wireless networks such as Ad-hoc Networks, there are some problems like not following balance for transmission of several data streams and also a through put reduction of flows.

These problems for using simultaneous flows in several parallel or intersect connection would occur in network topology. A connection based on congestion control in TCP would be followed by unbalance network. Resource limitations and Ad-hoc network radio interference require throughput and better and balanced performance.

Packet loss in two TCP interference causes TCP congestion control mechanism stimulation, unbalance, reduction in throughput and lower performance in the network. When web reviewer pages are consisted of several objects and transmission is done by a TCP connection, each transmission containing an object requires a complete waiting for previous transmission. In other words, when several simultaneous connections are used, there is a capability for transmitting some objects with a high speed through other simultaneous connections (even despite packet loss in a connection) which is called head-of-line Blocking (HOL) method. Although transmission by several simultaneous connections would cause a higher transmission speed, it could be followed by balance decrease and network delay time increase.

Another basic problem is route failure in Ad-hoc networks appeared by nodes movement causing TCP congestion control mechanism stimulation and as a result sever reduction of performance and excessive increase in TCP flow delay. Sending ack messages from one of permanent users of band width is wireless channel and naturally incase of collision in channel it would be doubled. The present discussion main goal is to provide an effective algorithm in As-hoc networks with high reliability and performance for data transmission comparing to former methods. To do this, SCTP protocol is used for implementing the suggested algorithm. The offered algorithm includes two parts: fix RTO and dynamic sack whose implementation is done by the help of NS-2 simulator and throughput parameters and packet loss delay are proposed in the from of standard diagrams, and ultimately are compared in the from of standard diagrams, and ultimately are compared with previous methods and the results are set forth. 


\subsection{Related Work}

In article (Doo-Won \& Hyuncheol, 2008) SCTP protocol has been investigated based on transmission management methods by multi-flow and observing fairness (flow balances) among these flows in SCTP connections. Article (Doo-Won \& Hyuncheol, 2008) seeks to offer a method for congestion control for various flows in SCTP by following the fairness of flows resulting in solving proposed problems in traditional SCTP method. In article (Kim \& Chung, 2007), SCTP protocol has been used as a reliable for delivering messages. In the mentioned article, a file transmission system of SCTP protocol has been applied by using multi-flow feature and congestion control for flows and the main objective is to solve line-blockage problem in transmitting several files and reduce load capacity of server. However, in this article, the suggested issues have been examined in fixed and unchanged network, requiring more discussion and investigation in other networks (wireless and Ad hoc networks) as well. In article (Al-Jubari, Othman, Mohd-Ali \& Hamid, 2011), challenges and solutions of the mentioned problems in Ad hoc multi hob network have been expressed by using TCP protocols. The crucial features of these networks, such as, mobility (movement), high rate of errors, asymmetric route, hidden and exposed nodes and dynamic routes cause inefficiency or mal-performance of SCTP protocol in this type of networks. In (Al-Jubari, Othman, Mohd-Ali \& Hamid, 2011) the main goal is to express vital challenges and to create a route for solving these challenges mentioning Fixed RTO as a method for reducing error rates and producing a difference between route failure and network congestion. This method is separated from other layers or protocols and do not need connection to lower layers for getting information about this issue; however, the exact and precise function of this method and the obtained results have not been stated. The fixed RTO method in article (Mohamed, Fisal \& Mohd, 2002) has been offered to solve pocket less problem because of Node replacement, a retransmission algorithm in transport protocol in SCTP protocol, which simulation results demonstrates performance optimization by using the new algorithm compared to the original TCP algorithm.

Furthermore, in (Fard, Bakar, Karamizadeh \& Foladizadeh, 2011), in capacity of TCP protocol in recognizing route failure or error and network congestion in mobile Ad hoc network has been tested in which a new method for end-to-end transport is offered. This method uses change record of queue rate to distinguish the difference or variance between route failure and congestion which sudden shifts in using queue and queue rate shows losses caused by congestion and moderate shifts based on queue application rate mean caused by route failure .

In (Al-Jubari \& Othman, 2010) Aljubari and etal, expressed weak characteristics of wireless channel as one of the main reasons for performance reduction of TCP protocol in multi bob wireless networks. In this article, transmitted pocket Acknowledge was introduced as one of the channel consumer main factors such as, extra data pockets in channel, able to reduce TCP protocol throughput. Moreover, another basic factor is collision of Acknowledge (response) and data pockets in the same route in a channel. In (Li, Lukyanenko, Tarkoma \& Yla-Jaaski, 2013) an adjustable delayed algorithm has been proposed, increasing TCP protocol performance significantly. In (chen \& Miller, 2008) Chen and etal pointed out TCP protocol performance decrease in multi hob wireless networks based on two factors such as congestion window and network traffic control based on incorrect use of band width or existing channel in network and took action to suggest solutions for increasing performance and throughput based on mentioned problems, including selective cumulative Acknowledge method in TCP protocol is improved and based on proposed simulations causes performance augmentation in the network. In article (Takemoto, Funasaka, Teshima, Ohta, \& Kakuda, 2009) Fixed RTO method and delayed selective Acknowledge are propounded implemented in Ad hoc networks using SCTP protocol. The mentioned method causes performance optimization in these type of networks, but these application performances and throughputs for Fix RTO are only calculated for good put(useful throughput in applications) which are mentioned method causes the lack of suitable result offer for investigating Fixed RTO algorithm performance. In Fixed RTO the rate of throughput and delay have a direct relationship with the number of transmitted pockets, if good put can compute transmitted pocket ratio correctly or reached to the target, without considering transmitted pockets on all of the transmitted pockets, which this point is not thought in article (Takemoto, Funasaka, Teshima, Ohta, \& Kakuda, 2009). Retransmission algorithm takes measure to set RTO and then retransmit pockets , therefore, to investigate the amount of performance optimization of this algorithm, throughput (the ratio of correct pockets to the target whether by one or several transmission on all of the transmitted pockets ) must be calculated. In this way, this algorithm precise performance amount can be computed.

In article (Takemoto, Funasaka, Teshima, Ohta, \& Kakuda, 2009) Take Moto etal, used selective Acknowledge algorithm for solving pocket collision problems in the route, which this algorithm provides the possibility of several Acknowledge (response) transmission for one pocket based on existing pockets in channel. In addition, the proposed algorithm, calls the need for re-examination of closed timer while cumulative Acknowledge pockets are transmitted and when transmission is occurring, the time between pockets and timers should be 
calculated again, and after each calculation, selective Acknowledge pockets must be tested and produced. In article (Takemoto, Funasaka, Teshima, Ohta, \& Kakuda, 2009) only good put was investigated based on this algorithm and it is implemented in Qual Net simulator, and it requires more precise examination on throughput and pocket delay grounds.

\section{Suggested Problems for Transmission Protocols in Ad-Hoc Networks and Offered Solutions}

Some of the mobile ad-hoc network problems related two SCTP and TCP transport protocol are propounded and investigated in this section (Al-Jubari, Othman, Mohd-Ali \& Hamid, 2011; Arun, Lokanatha, Reddy \& Hiremath, 2008; Hurtig \& Brunstrom, 2008; Ghosekar, Pravin, Girish \& Pradip, 2010):

\subsection{Network Throughput in Multi Connection}

TCP protocol for data transportation is designed through single connection, then for simultaneous data transmission multi connection TCP are needed. Total amount or size of multi connection congestion window is greater than the size of single connection congestion window which is because of TCP congestion control mechanism performance based on one connection, causing lack of balance and performance in an ad-hoc-network. Furthermore, if a service uses one connection and its equivalent service uses multi or several connections, throughput of these two services will be different causing an unfair division or share of routing capacity (Rüngeler, Tüxen \& Rathgeb, 2009).

The suggested solution in the article (Doo-Won \& Hyuncheol, 2008; Ansari, Rahman \& Shukla, 2012; Tasneem, Yunus \& Kanpurwala, 2006)for this issue is to use SCTP protocol, since this protocol includes a mechanism called transmission by multi-streaming for several simultaneous transmission through one SCTP association. As a result, SCTP congestion control functioning again based on one association does not have a problem.

Comparing TCP flow with SCTP flow performances is done by simulator and it is illustrated in fig-1 for better description. According to fig-1, flow 1 is transmitted from zero node to node seven and flow 2 is sent from node 1 to node 8 and connection link is shared from node 2 to node 6 . It is worth mentioning that only one set of data, i.e. only one data stream is sent by flow 1 . In order to investigate these two flows performances, the two flow throughput is calculated by various data rates and the obtained results are demonstrated in fig-2. In other words, more increase in the number of connections or data streams in a STCP flow, make possible the throughput confirmation and augmentation for flows. However, TCP flow 1 contain only one connection, not able to divide bandwidth fairly and flow 2 acquires more share of bandwidth.

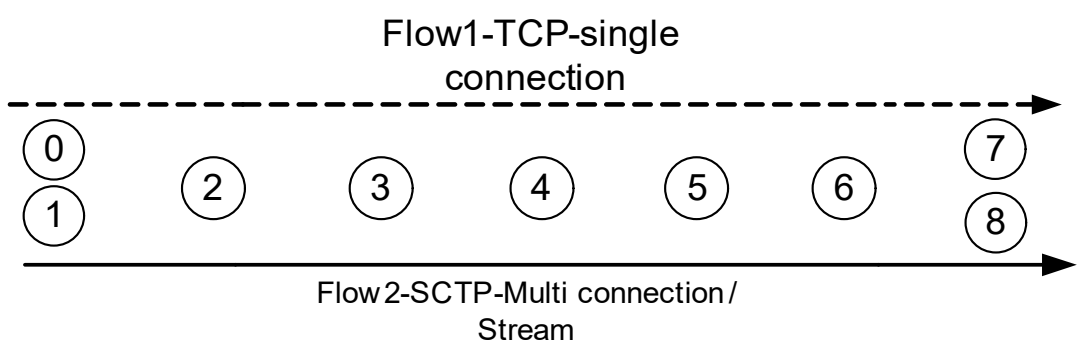

Figure 1. First simulation topology by using flow transmission having one or several associations

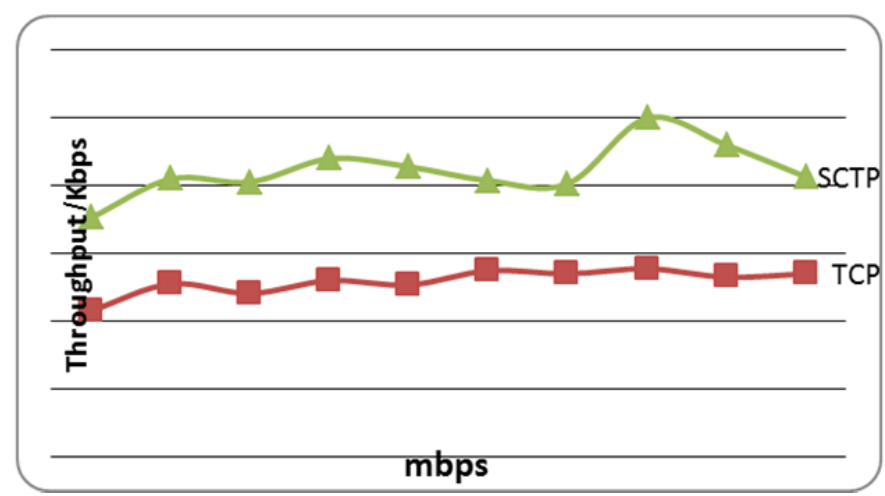

Figure 2. Network throughput rate for TCP, SCTP and SCTP Protocols 


\subsection{The Problem of Route Failure Because of Nodes Movements}

When node movement are concerned, it is necessary to consider this point that routing algorithms in forming a route and re-establishing a connection when node movement occurs may cause a problem and as a result, the previous formed route for transmission will failed. This problem causes an asymmetric route in transmission. When round trip route of data is similar, the above problem merely causes a change route, or there will be asymmetry in route. The amount of this asymmetry is related to routing protocol.

To face with the problem, transport layer protocols attempt to re-send packets and increase the amount of congestion control window. This condition excess with the high amount of nodes movements, and because of separate route existence for sending and respond, route failure will increase in TCP connection and as a result, delay in identification and re-formation of route will cause throughput reduction and excessive connection delay. It is necessary to note that both TCP and SCTP protocols have a similar method for back off RTO process to avoid congestion and cause RTO increase. In sending a packet to a destination address, if ack packet in timer time limit is not sent to sender or is received after the end of confirmation response((ack packet) timeout, the time of RTO will be doubled comparing to previous amount(Shi, Jin, Guo \& Cheng , 2004). This mechanism in ad-hoc networks cause an increase in sender waiting time and lead to an increase in packet delay time.

\subsection{Packet-Collision in a Wireless Channel}

Packet-collision probability in wireless networks is proportionate to the number of nodes and because of excessive packet collisions, network throughput will decrease severely (Sengottaiyan, Somasundaram \& Arumugam, 2010). In this situation, packet-collision detection will not perform effectively. Then another method called packet-collision avoidance will be applied. In collision-detection method, sender node wait for an event and after its occurrence announce collision, but in collision-avoidance method, the transmission speed is reduced to a restricted amount. This method similar to limiting speed in highways during rush-hours considered to prevent accidents. However, in other method one is waiting for a collision and then he has to reduce the speed suddenly. Continual usage of collision-avoidance method causes reduction of useful maximum band-width and makes band-width to be available less than the needed amount. Eventually, this ends to a network performance and connection channel decrease, and for this reason, the mentioned method application is recommended only in specific conditions.

In transport layer, packet-collision control, packet loss and flow control are due to transport layer protocols such as TCP and SCTP. These protocols contain mechanisms such as congestion control and flow control whose responsibility is to control network traffic and data transport reliability.

Nodes use acknowledgement packet for transmitted messages responses and these packets are transported in apposite direction with a shared wireless channel, so cause a collision increase in the channel.

\subsection{The Proposed Method for SCTP Protocol Improvement in Mobile Ad Hoc Networks}

The present study offers the two following algorithms for solving problems:

\subsubsection{Fixed RTO}

The amount of fixed RTO is fixed in case of packet lass, and this fixed amount, on the condition that sender succeeds to re-transmit the packet properly, RTO control will return to its former condition.

After the first packet transmission, the sender waits for message acknowledgment packet from receiver which is called the amount of time between packet transmission and packet acknowledgment receive (Round trip time). In fixed RTO algorithm, the amount of RTO is fixed and only if a packet of message acknowledgment by sender, the amount of RTT will be calculated by which fixed RTO is measured. The model of calculation is as the following formula (Mohamed, Fisal \& Mohd, 2002):

$$
\mathrm{FxRTO}=\mathrm{RTT} * \mathrm{p}
$$

According to the above formula $\mathrm{p}$ is a fixed amount between 3 and 4, and the amount of FxRTO has a direct relationship with the early calculated RTT. As it is obvious from this simple formula it is possible to analyze the network even at the first packet transmission. In this paper $\mathrm{p}$ is assumed to be equal to 3.5 in this way, rather than waiting to detect network problem (rout change or establishing a new rout), it can be possible to fix the maximum number of re-transmissions to avoid waste waiting time and to prevent an increase in probability of congestion occurrence and to refuse network throughput reduction, as a result reach to an efficient performance. 
The following pseudo-code express as a method for using fixed RTO in SCTP:

\begin{tabular}{|c|c|}
\hline $\begin{array}{l}\text { Function Rtt update } \\
\text { Begin } \\
\text { If Rtt measurment is True then } \\
\text { Begin } \\
\text { If flag is true then } \\
\text { Begin } \\
\text { Set RTO=save_RTO * } p \\
\text { Set flag }=0 \\
\text { End } \\
\text { Else } \\
\text { Begin } \\
\text { Set Rtt measurment }=\text { false } \\
\text { End } \quad \cdots \cdots \\
\text { End } \quad \\
\text { If flag }=1 \text { then } \\
\text { Begin } \\
\text { Set RTO }=\text { save_RTO* } p \\
\text { End } \\
\text { End Function }\end{array}$ & $\begin{array}{l}\text { Function Timeout } \\
\text { Begin } \\
\text { If flag is not } 1 \text { and timeout_count }>1 \text { then } \\
\text { Begin } \\
\text { set flag }=1 \\
\text { set save_RTO=RTO } \\
\text { set RTO }=\text { RTO* } p \\
\text { End } \\
\text { Else } \\
\text { Begin } \\
\text { Set flag }=0 \\
\text { End } \\
\text { End Function }\end{array}$ \\
\hline
\end{tabular}

In the above code, the probability of packet loss is announced with the completion of timer time. When a packet is lost in connection route and is detected by the sender, the Timeout function will be activated. In this function, first, it is investigated whether the amount of RTO has been fixed before, or not, and whether the time of timer has reached to the end or not, (through examining the amount of variable "i Timeout count"). In case of establishing conditions, the sign related RTO will change and the amount of RTO for a mode which packet is re-transmitted, will be saved. Eventually with multiplying RTO by $p$, FxRTO will be obtained. Other wise it return to its regular mode and uses timer previous conditions.

\subsection{2 "Dynamic Delayed Selective Acknowledgment Algorithm" for Ad Hoc Networks}

This algorithm is based on response using "SACK" for data chunk. If next packet ready to transmit, contains several sequential orderly number, a cumulative ack delayed message can be produced and confirm several packet receive at the same time. This acknowledgment message is at least produced for two packets and ultimately it causes a reduction in the number of SACK message and reduces collision.

In figure - 3,a sample of this method based on a response for two packets has been shown, which this mode in SCTP protocol is defined by DELAYED-SACK-TRIGGER variable which has the primary amount equal to 2 similar to fig-3.

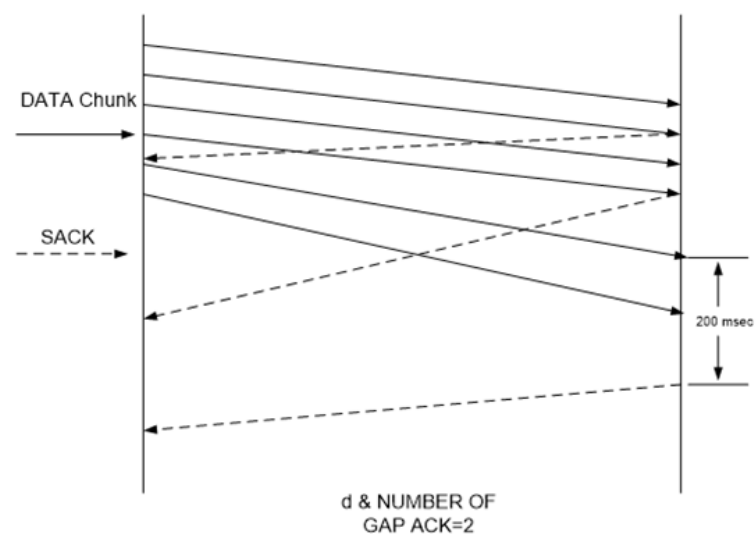

Figure 3. Dynamic Delayed selective acknowledgment performance 
Figure 3. is an example of Dynamic Delayed Selective Acknowledgment method for transmitting two packets confirmation. When the second packet doesn't receive properly and $200 \mathrm{~ms}$ (obtained early RTO) from receiving one packet has passed, nly one selective acknowledgment packet (SACK) for confirming the first packet will be transmitted.

The improved or optimized algorithm is as the following:

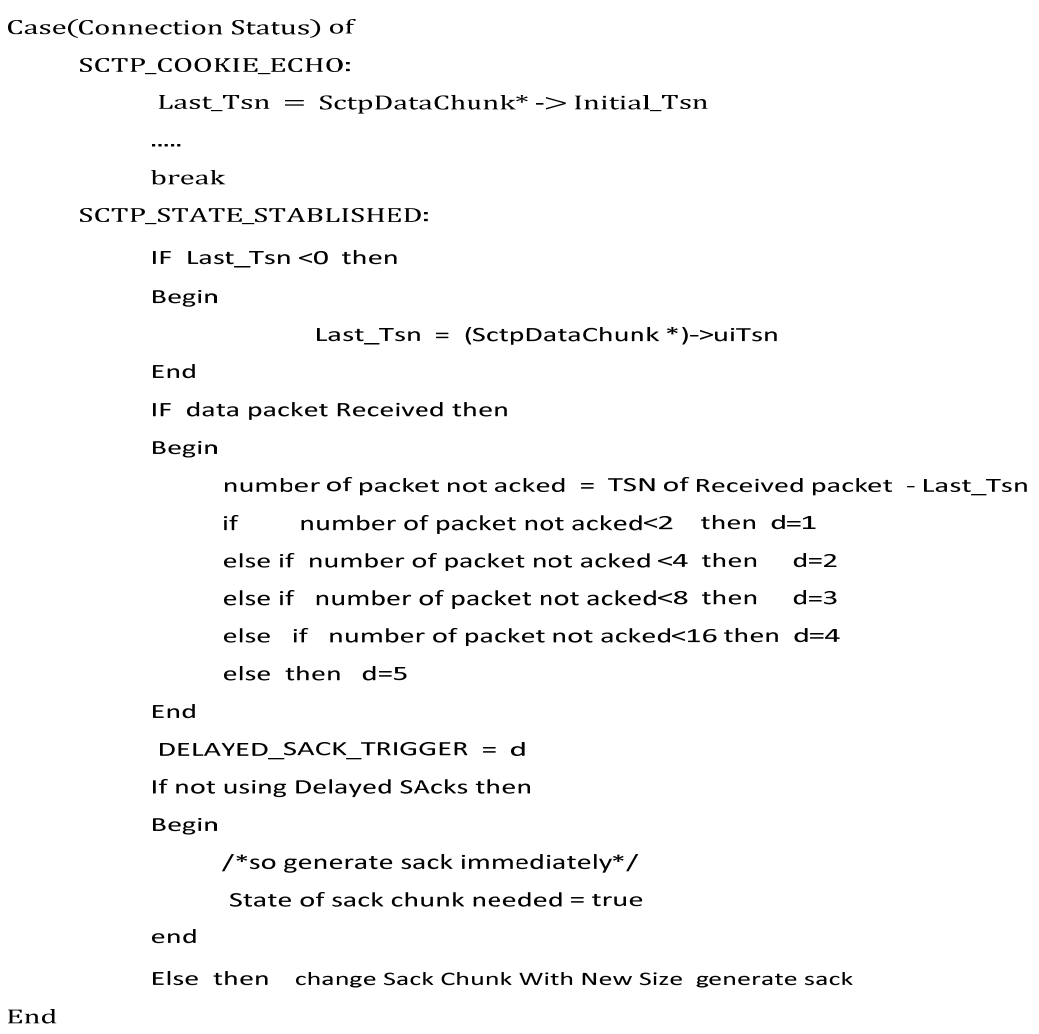

The above proposed algorithm expresses the suggested method for acknowledgment with dynamic delay in mobile ad-hoc network. In this algorithm first transfer sequence number will be saved (Base-TSN) and in stabilized condition of connection if a packet is received, the number of packets which has not yet been confirmed can be obtained by using the difference between first saved sequence number and received packet transfer sequence number. By using this method the number of packets (d) which should be accompanied by SACK packet will be detected.

On receiver side, the time of receiving previous packet will be saved and is compared to previous packet round-trip time amount and if this amount is more than the thresh hold amount which is detected and the received packet includes cumulative transfer sequence number, then Base-Tsn will be updated or upgraded to the new transfer sequence number. Therefore, transmission reduction of acknowledgment packet causes packet collision probability reductions in a wireless connection channel.

The $\mathrm{p}$-value can be obtained based on the following equation and d-value that $p$-value is calculated dynamically:

\section{Simulation}

$$
p=3.3+\mathrm{d}^{*} 0.05
$$

For investigating the rate of the two suggested algorithm effectiveness, in this research, SCTP and TCP and proposed protocols will be assessed by using NS-2 simulator. Simulating is examined by using three various topology. For every topology, there are two diagram illustrated to express how through put average amounts are described by a table. Three protocols such as proposed SCTP(improved protocols based on proposed algorithms), SCTP, TCP were investigated.

\subsection{The First Topology Simulation}

In the first topology, the grid network topology demonstrated in fig-4 will be used (Scheuermann, Lochert \& Mauve, 2008). This topology uses 802-11 and AODV protocols in NS-2 simulator (Takemoto, Funasaka, 
Teshima, Ohta, \& Kakuda, 2009; Suvarna, Kishore, Parimala \& Kumar, 2014). The simulation time is 300 seconds and data rate is 11 Mega bit in second and transmission or data boundary is $250 \mathrm{~m}$.

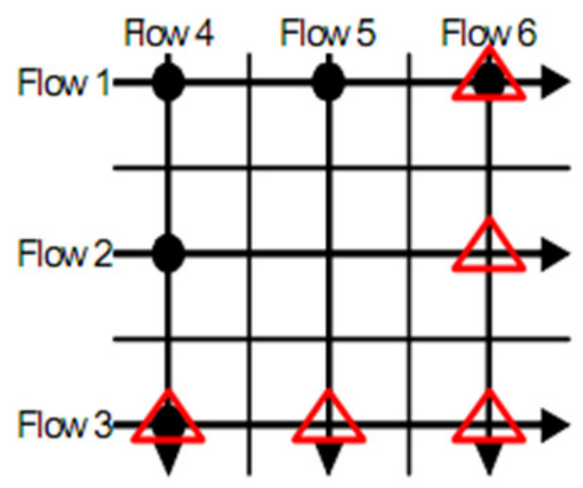

Figure 4. Grid topology

The data stream for 3,2,1 flows is equal to 2 and the data stream for 6,5,4 flows is equal to 1 . Every flow throughput average amount for the applied protocols in simulation has been demonstrated fig-5. moreover, the transmitted packet delays in the present topology has been shown in fig-6.

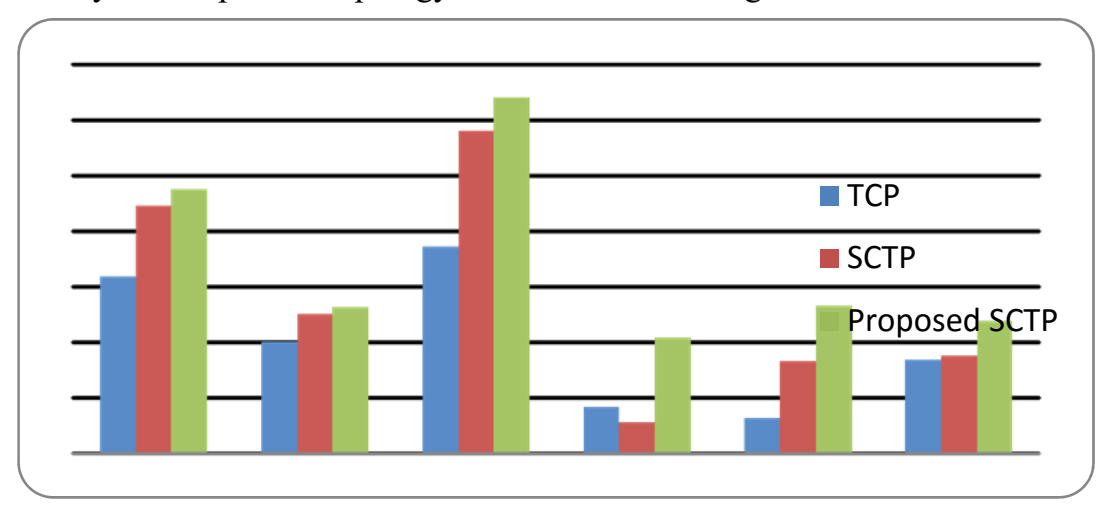

Figure 5. Flow throughput average in Grid topology

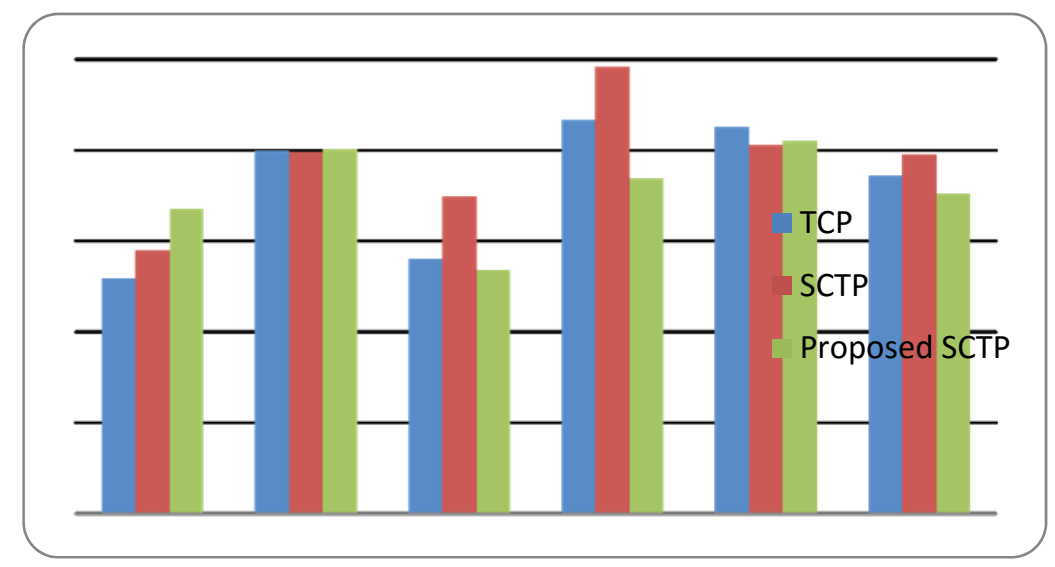

Figure 6. Packet delays for every data stream in Grid topology

As it is clear in fig-5, the proposed protocol throughput was better and throughput average was dominated comparing to two other protocols according to table- 1 and it is optimized to nearly $20 \%$. Furthermore, this superiority is very significant in packet delay time. 
Table 1. Throughput average and packet delays for all of the flows in various protocols in the Grid topology

\begin{tabular}{llll}
\hline Proposed SCTP & SCTP & TCP & Protocol \\
\hline 1720 & 1401 & 1006 & Avg Throughput [Kbps $]$ \\
178 & 195 & 181 & Avg packet delay (ms) \\
\hline
\end{tabular}

\subsection{The Second Topology Simulation (Mobile Ad-Hoc Networks)}

The second simulation based on mobile ad-hoc network topology is designed in fig-7. In this environment, there are 50 nodes in a space of $300 * 1200$ square meter. In this environment, there are 6 nodes as sender-receiver, 3 of them are as source and the other 3 nodes are as destination which are considered to be fixed. Also the rest of the nodes are moving randomly in this environment and the number of data transmission flows is equal to 3 . In this topology the three protocols such as; TCP, SCTP and protocols SCTP are under investigation by using AODV routing protocol. In fig-8, throughput and in fig-9, packet delay times for this topology in the presence of three mentioned protocols and three flows have been demonstrated, which this graph expressing the superiority of proposed method to previous protocols.

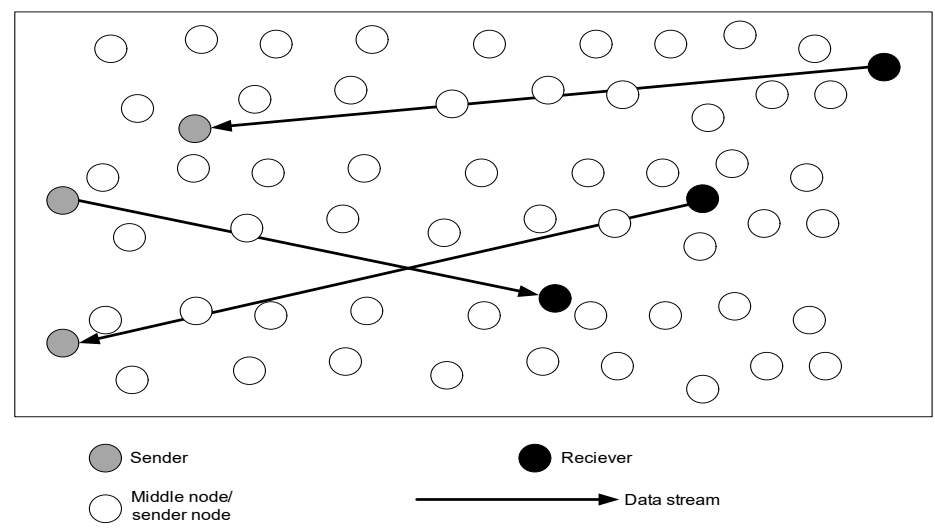

Figure 5. The second topology for mobile ad-hoc network

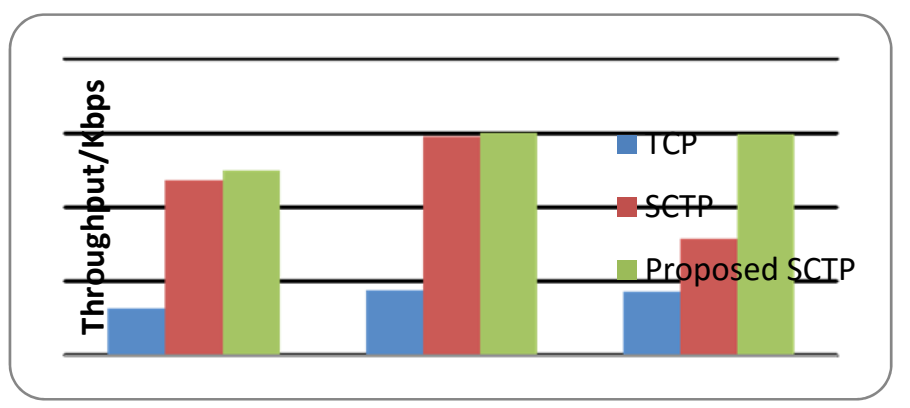

Figure 8 . The flow throughput average by using TCP, SCTP, Proposed SCTP in mobile ad-hoc network topology (second topology)

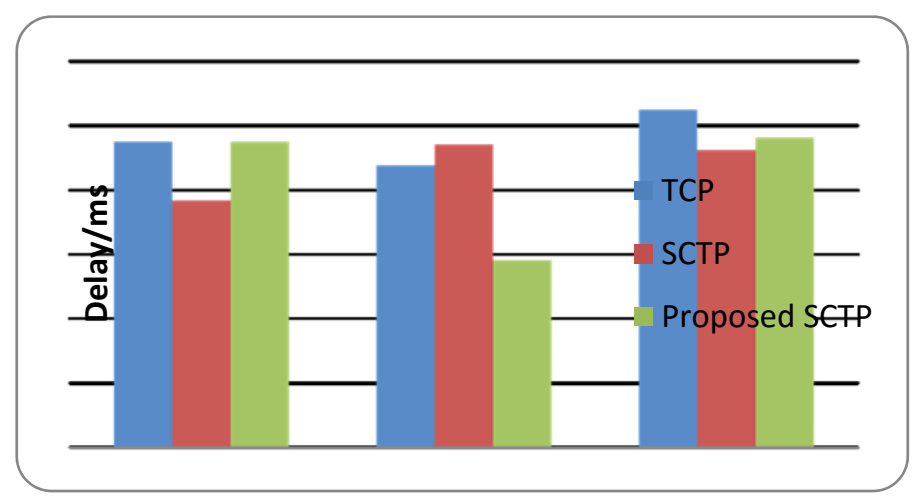

Figure 9. Packet delays for every data stream in Mobile Ad-Hoc Network topology (second topology) 
In Table 2, throughput and packet delay averages in flows are explained for every three protocols. In this topology, considering the movement of the nodes, it can be observed that the routs change alternatively, then the second part of proposed algorithm, that is, dynamic delayed selective acknowledgment cannot act properly in sending respond packets with appropriate window size. However, the other part of algorithm, i.e. fixed re-transmission time acts properly and causes throughput increase and moreover, packet delay reductions. In addition, node movements should be considered moving randomly without any special pattern, but again, algorithm attempts to control transmissions and start re-transmission and with doing so assists to increase performance and efficiency. This scenario provides $20 \%$ throughput optimization and also $5 \%$ delay reduction.

Table 2. Throughput average and packet delays for all of the flows in various protocols in the second Topology

\begin{tabular}{llll}
\hline Proposed SCTP & SCTP & TCP & Protocol \\
\hline 1451 & 1151 & 397 & Avg Throughput [Kbps] \\
207 & 219 & 239 & Avg packet delay(ms) \\
\hline
\end{tabular}

\subsection{The Third Topology Simulation}

In this topology, an environment similar to the second topology was used. An environment with data rate of 11 megabit, 1200*300 square meter space or area, AODV routing protocol, containing 50 nodes which 3 nodes are fixed to one node in other part of network, transmit data stream. In this simulation for transmission, there data streams having single-flow are used. Fig-10 demonstrates the third topology.

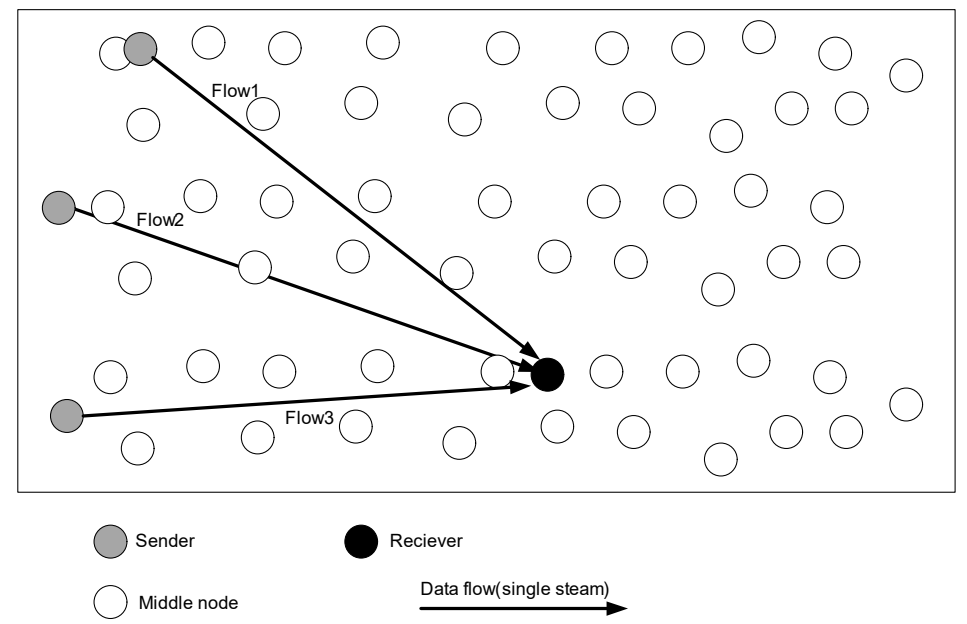

Figure 10. The third topology for mobile ad-hoc network

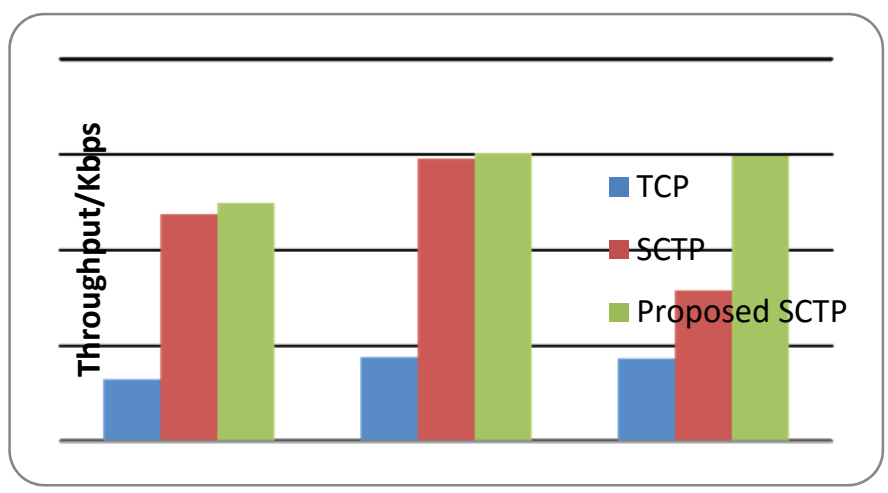

Figure 11. The flow throughput average by using TCP, SCTP, Proposed SCTP in third topology

In Figure 11. a comparative graph for three flows in the three protocols has been illustrated which the effect of transmission for one target or destination is calculated and demonstrated. 


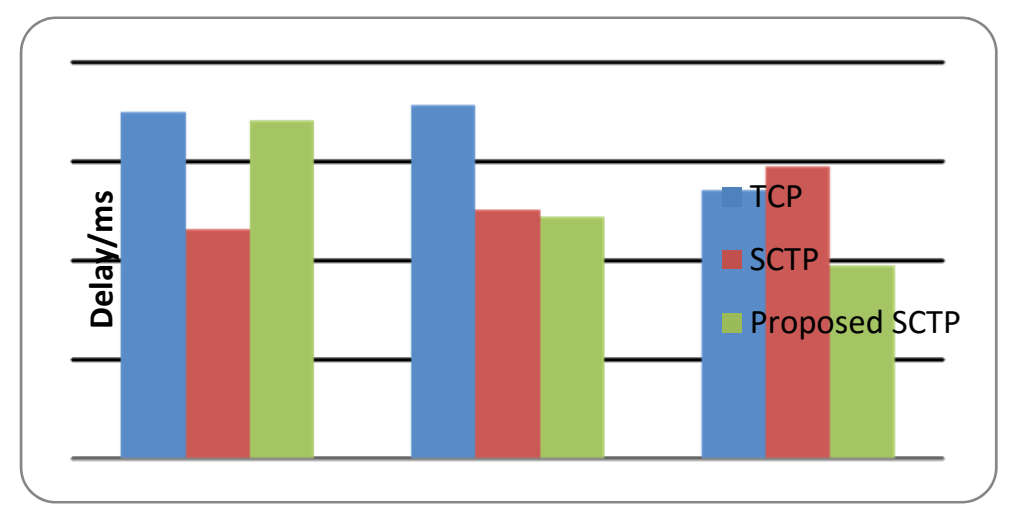

Figure 12. Packet delays for every data stream third topology

In table-3, also delay and throughput averages for this topology are calculated and stated. As it is obvious from the table, the proposed algorithm performance has been optimized based on delay and throughput.

Table 3. Throughput average and packet delays for all of the flows in various protocols in the third Topology

\begin{tabular}{llll}
\hline Proposed SCTP & SCTP & TCP & Protocol \\
\hline 601 & 555 & 262 & Avg Throughput [Kbps] \\
207 & 219 & 239 & Avg packet delay(ms) \\
\hline
\end{tabular}

In Figure 12. packet delays in the network are computed for every flow and each three protocols and were illustrated based on the graph.

\section{Conclusion}

Considering nature and the type of source limitations in ad-hoc networks and problems such as route failure and an increase in congestion, there is a need for exact and precise management technique for allocation and consumption of resource. This method provide a possibility to control transmissions in the network with regard to replacement change. In the present article two algorithms of foxed RTO and selective acknowledgment are suggested dynamically, which solves the mentioned problems. According to the offered graphs and diagrams in this research and the obtained amounts and quantities, it can be concluded that the proposed method is not only appropriate to use in ad-hoc networks but it is also useful for mobile networks. Furthermore, the use of the two mentioned methods in SCTP protocol, i.e. using RTO control with a fixed coefficient and selective acknowledgment number control, cases superiority and preference of the proposal method with respect to the two other protocols. This suggested method causes packet delay time-reduction and prevents a time-increase for re-transmission because of retransmission time control and it searches to use better of connection links.

\section{References}

Stewart, R., \& Xie, Q., Morneault, K., Sharp, C., Schwarzbauer, H., Taylor, T., Rytina, I., Kalla, M., Zhang, L., \& Paxson, V. (Oct. 2007). Stream Control Transmission Protocol. RFC4960.

Chen, J. M., et al. (2011). Improving SCTP Performance by Jitter-Based Congestion Control over Wired-Wireless Networks. EURASIP Journal on Wireless Communications and Networking, 1-13.

Kim, H., Kim, Y., Kim, K., \& Chung, J. (2007). High-performance data transfer using SCTP-based compact association scheme. International Conference on Computational Science and its Applications, ICCSA, 389-398.

Al-Jubari, A. M., Othman, M., Mohd-Ali, B., \& Abdul-Hamid, N. A. (December 2011). TCP performance in multi-hop wireless ad hoc networks: Challenges and solution. EURASIP Journal on Wireless Communications and Networking.

Mohamed, Y., Fisal, N., \& Mohd, A. (2002). Performance of TCP on Mobile IP network during handoffs. Student Conference on Research and Development(SCORD), 390-393. 
Fard, M. A. K., Bakar, K. A., Karamizadeh, S., \& Foladizadeh, R. H. (May 2011). Improve TCP performance over mobile ad hoc network by retransmission timeout adjustment. 3rd International Conference on Communication Software and Networks (ICCSN), 437-441.

Al-Jubari, A. M., \& Othman, M. (2010). A new delayed ACK strategy for TCP in multi-hop wireless networks. International Symposium in Information Technology (ITSim), 2, 946-951.

Li, M., Lukyanenko, A., Tarkoma, S., \& Yla-Jaaski, A. (2013). The Delayed ACK evolution in MPTCP. In Global Communications Conference (GLOBECOM), IEEE, 2282-2288.

Chen, B., Marsic, I., \& Miller, R. (2008). Issues and Improvements in TCP Performance over Multihop Wireless Networks. Sarnoff Symposium, IEEE.

Takemoto, Y., Funasaka, J., Teshima, S., Ohta, T., \& Kakuda, Y. (2009). SCTP performance improvement for reliable End-to-end communication in ad hoc networks. International Symposium on Autonomous Decentralized Systems, 1-6.

Arun, K. B. R., Lokanatha, C., Reddy, P., \& Hiremath, S. (2008). MOBILE AD HOC NETWORKS: ISSUES, RESEARCH TRENDS AND EXPERIMENTS. International Engineering \& Technology (IETECH) Journal of Communication Techniques, 2.

Ghosekar, P., Girish, K., \& Pradip, G. (2010). Mobile ad hoc networking: imperatives and challenges. IJCA Special Issue on MANETS, 153-158.

Rüngeler, I., Michael, T., \& Erwin, P. R. (2009). Congestion and flow control in the context of the message-oriented protocol SCTP. NETWORKING 2009 Springer Berlin Heidelberg, 468-481.

Tasneem, Y. K. (2006). SCTP multi-streaming: Study of transmission of partially reliable and reliable data using dynamic stream priorities. The Degree of MASTER of SCIENCE, Oklahoma State University.

Shi, J. Y., Jin, Y. H., Guo, W., \& Cheng, S. D. (March 2004). Performance evaluation of SCTP as a transport layer solution for wireless multi-access networks. Wireless Communications and Networking Conference, 1 , 453-458.

Doo-Won, S., Hyuncheol, K. (April 2008). Design of SCTP-SCF",IJCSNS International Journal of Computer Science and Network Security, 8(4).

Scheuermann, L. M. (April 2008). Implicit hop-by-hop congestion control in wireless multihop networks", Ad Hoc Networks, 6(6), 60-286.

Per, H., \& Anna, B. (October 2008). Enhancing SCTP loss recovery: An experimental evaluation of early retransmit. Computer Communications, Performance Evaluation of Communication Networks, 31(25).

Boussen, S. N., \& Tabbane, S. (Nov. 2009). Performance analysis of SCTP protocol in WiFi network. First International Conference on Communications and Networking, IEEE, pp. 1-5, 3-6

Sengottaiyan, N., Somasundaram, R., \& Arumugam, S. (Oct. 2010). A Modified Approach for Measuring TCP Performance in Wireless Adhoc Network. International Conference on Advances in Recent Technologies in Communication and Computing (ARTCom), 267 - 270, 16-17.

M.ansari, S. A., \& Rahman, U. S. (March 2012). SCTP Protocol Reviews and Performance in Ad-Hoc Multi-Homed Networks. International Journal of Advanced Research in Computer Science and Software Engineering, 2.

Mbarushimana, C., \& Shahrabi, A. (May 2013). Alleviating contention-induced spurious timeouts in QoS-aware MANETs. Ad Hoc Networks, 11, 879-893.

Suvarna, K., \& Parimala, K. (Feb. 2014). Performance Estimation of DSR, DSDV and AODV in TCP, UDP and SCTP. IEEE International Conference on Optimization, Reliabilty and Information Technology (ICROIT), 195-198, 6-8.

\section{Copyrights}

Copyright for this article is retained by the author(s), with first publication rights granted to the journal.

This is an open-access article distributed under the terms and conditions of the Creative Commons Attribution license (http://creativecommons.org/licenses/by/3.0/). 